\title{
Stability Analysis of a Harvested Prey-Predator Model with Stage Structure and Maturation Delay
}

\author{
Chao Liu, ${ }^{1,2}$ Qingling Zhang, ${ }^{1,2}$ and James Huang ${ }^{3}$ \\ ${ }^{1}$ Institute of Systems Science, Northeastern University, Shenyang, Liaoning 110819, China \\ ${ }^{2}$ State Key Laboratory of Integrated Automation of Process Industry, Northeastern University, Shenyang, Liaoning 110819, China \\ ${ }^{3}$ Department of Applied Mathematics, Hong Kong Polytechnic University, Hong Kong
}

Correspondence should be addressed to Chao Liu; singularsystem@yahoo.com.cn

Received 7 February 2013; Accepted 11 April 2013

Academic Editor: Ligang Wu

Copyright (C) 2013 Chao Liu et al. This is an open access article distributed under the Creative Commons Attribution License, which permits unrestricted use, distribution, and reproduction in any medium, provided the original work is properly cited.

\begin{abstract}
A harvested prey-predator model with density-dependent maturation delay and stage structure for prey is proposed, where selective harvest effort on predator population is considered. Conditions which influence positiveness and boundedness of solutions of model system are analytically investigated. Criteria for existence of all equilibria and uniqueness of positive equilibrium are also studied. In order to discuss effects of maturation delay and harvesting on model dynamics, local stability analysis around all equilibria of the proposed model system is discussed due to variation of maturation delay and harvest effort level. Furthermore, global stability of positive equilibrium is investigated by utilizing an iterative technique. Finally, numerical simulations are carried out to show consistency with theoretical analysis.
\end{abstract}

\section{Introduction}

In the natural world, many species have a life history that takes them through two stages, juvenile stage and adult stage. Individuals in each stage are identical in biological characteristics, and some vital rates (rates of survival, development, and reproduction) of individuals in a population almost always depend on stage structure. Furthermore, many complex biological phenomena arising in prey-predator ecosystem always depend on the past history of system, and it has been recognized that time delay may have complicated impact on dynamics of prey-predator ecosystem [1]. In the past several decades, there has been an increasing interest in prey-predator model system with stage structure and time delay (see [2-26] and the references therein).

In the model proposed by Aiello and Freedman [2], stage structure of single population growth with stage structure and time delay representing for maturation of population is considered. Their model predicts a positive steady state as the global attractor, thereby suggesting that stage structure does not generate sustained oscillations frequently observed in single population in the real world. Subsequent work made by other authors $[3,6,7,12-14]$ suggests that time delay to adulthood should be state dependent. Generally, boundedness and persistence of solutions of model system may be affected by introduction of time delay into preypredator system with stage structure $[14,15,20-22,24-$ 26]. Time delay can also cause loss of stability and other complicated dynamical behavior [27]. Especially, there is a well-developed theory of stage-structured models which incorporate time delay into maturity of population [4].

It is well known that harvesting has a strong impact on dynamic evolution of a population; there has been considerable interest in the modeling of harvesting of biological resources [1]. In these models, the harvesting effort is considered to be a dynamic variable; several kinds of harvesting policies are utilized to study the dynamical behavior of the model system. In recent years, there has been growing interest in the study of stage-structured prey-predator system with harvesting. Several prey-predator models with stage structure and harvest effort on predator have been investigated in [2833 ] and the references therein. 
Recently, Huo et al. [24] investigated dynamical behavior and stability of the following stage-structured system with time delay:

$$
\begin{gathered}
\dot{x}_{1}(t)=r_{1} x_{2}(t)-d x_{1}(t)-r_{1} e^{-d \tau} x_{2}(t-\tau), \\
\dot{x}_{2}(t)=r_{1} e^{-d \tau} x_{2}(t-\tau)-b x_{2}^{2}(t)-\frac{a_{1} y(t) x_{2}(t)}{x_{2}(t)+k_{1}}, \\
\dot{y}(t)=y(t)\left(r_{2}-\frac{a_{2} y(t)}{x_{2}(t)+k_{2}}\right),
\end{gathered}
$$

where $x_{1}(t), x_{2}(t)$, and $y(t)$ represent the density of immature prey population, mature prey population and predator population, at time $t$, respectively; $r_{1}$ is the intrinsic growth rate of mature prey population, and $d$ is the death rate of immature prey population. Constant $\tau \geq 0$ denotes maturation delay of immature prey population to mature prey population, and the term $r_{1} e^{-d \tau} x_{2}(t-\tau)$ represents the immature prey population who were born at time $t-\tau$ and survived at time $t . b$ denotes the intracompetition rate for mature prey population due to overcrowding phenomenon with mature prey population. $a_{1}$ is the maximum value of the per capita reduction rate of mature prey population due to predator population, and $a_{2}$ is the maximum value of the per capita reduction rate of predator population due to mature prey population. $k_{1}$ measures the extent to which the environment provides protection to mature prey population, and $k_{2}$ measures the extent to which the environment provides protection to predator population. $r_{2}$ represents the maximal per capita growth rate of predator population. All the parameters mentioned previously are all positive constants. Furthermore, global stability of positive equilibrium of model system (1) is investigated in [26].

It is well known that the length of time for prey population to maturity is density dependent; that is, maturation time depends on the total population amount of prey population within prey predator ecosystem, and prey population takes less time to reach maturity with depletion of predator population [23,34-36]. Density-dependent maturity of population in prey predator ecosystem is discussed in their work, which reveals that density-dependent effects of the predators' counterparts to prey defenses and the density dependence effect of each type of predator offense are analogous to the corresponding type of prey defense. Dynamical behavior and stability switch is investigated in [23, 34-36]. However, harvest effort on population within prey-predator ecosystem is not considered in [23, 34-36].

By assuming maturity delay of prey population is density dependent and predator population is harvested; work done in [24] is extended in this paper, and a harvested prey predator model with density-dependent maturation delay and stage structure for prey population is proposed in the second section of this paper. In the third section of this paper, positiveness and boundedness of solution of the proposed model are studied, and the conditions for existence of equilibria and uniqueness of positive equilibrium are also investigated. Local stability analysis around all equilibria is discussed due to variation of maturation delay as well as harvest effort level. Furthermore, global stability of the positive equilibrium of the proposed model system is studied by utilizing an iterative technique. In the fourth section of this paper, numerical simulations are carried out to show consistency with theoretical analysis. Finally, this paper ends with a conclusion.

\section{Model Formulation}

Based on the previous analysis, the model proposed by Huo et al. in [24] is extended by incorporating harvest effort on predator population and assuming that maturation delay of prey population is density dependent, and the model can be governed by the following differential equations:

$$
\begin{gathered}
\dot{x}_{1}(t)=r_{1} x_{2}(t)-d x_{1}(t)-r_{1} e^{-d \tau(z(t))} x_{2}(t-\tau(z(t))), \\
\dot{x}_{2}(t)=r_{1} e^{-d \tau(z(t))} x_{2}(t-\tau(z(t)))-b x_{2}^{2}(t)-\frac{a_{1} y(t) x_{2}(t)}{x_{2}(t)+k_{1}}, \\
\dot{y}(t)=y(t)\left(r_{2}-\frac{a_{2} y(t)}{x_{2}(t)+k_{2}}\right)-q E y(t) .
\end{gathered}
$$

The initial conditions for model system (2) take the following form:

$$
\begin{gathered}
x_{1}(0)>0, \quad y(0)>0, \\
x_{2}(\theta)=\phi(\theta)>0, \quad \theta \in[-\widehat{\tau}, 0),
\end{gathered}
$$

where $z(t)=x_{1}(t)+x_{2}(t)+y(t)$, a scalar $E \geq 0$ denotes the harvesting effort to predator population, constant $q$ is the catchability coefficient of predator, and the harvesting term $q E y(t)$ follows the catch per unit effort hypothesis [1]. Furthermore, $r_{1}, r_{2}, d, b, a_{1}, a_{2}, k_{1}$, and $k_{2}$ in model system (2) share the same interpretations mentioned in model system (1).

In the following section of this paper, model system (2) is derived under the following hypotheses.

(H1) Prey population is divided into two-stage groups, that is, immature and mature. The term $r_{1} e^{-d \tau(z(t))} x_{2}(t-$ $\tau(z(t)))$ represents the immature prey population born at time $t-\tau(z(t))$ and survive at time $t$ with death rate $d$, which represents transformation term from immature prey to mature prey.

(H2) Density-dependent time delay $\tau(z(t))$ is taken to be an increasing differentiable bounded function of the total population (immature prey, mature prey, and predator population), which satisfies

$$
\begin{gathered}
\frac{d[\tau(z(t))]}{d t} \geq 0, \quad 0 \leq \tau_{0} \leq \tau(z(t)) \leq \tau_{1}, \\
\lim _{z(t) \rightarrow 0+} \tau(z(t))=\tau_{0}, \quad \lim _{z(t) \rightarrow+\infty} \tau(z(t))=\tau_{1} .
\end{gathered}
$$

(H3) For the continuity of initial conditions, it is required that

$$
x_{1}(0)=\int_{-\widehat{\tau}}^{0} r_{1} e^{d s} \phi(s) d s
$$


where $\phi(s)$ is assumed to be continuous function (for mathematical reason) and nonnegative (for biological reason).

(H4) In order to exclude the possibility of immature prey becoming mature prey except by birth, $t-\tau(z(t))$ is assumed to be a strictly increasing function of $t$. Otherwise, there are two different times at which the same individual immature prey turns to be mature prey twice at the same instant of time, which is absurd to practical biological interpretations. (For detailed methodology, see [3].)

\section{Qualitative Analysis of Model System}

In this section, positiveness and boundedness of solution of model system (2) are analytically investigated. Criteria for existence of equilibria and uniqueness of positive equilibrium are also studied. By using differential dynamical system theory and stability theory, local stability analysis around all equilibria of model system is discussed. Furthermore, global stability of the positive equilibrium of the proposed model system is studied by utilizing an iterative technique.

\subsection{Positiveness and Boundness of Solutions}

Theorem 1. Under hypotheses (H1)-(H4), solutions of model system (2) with given initial conditions are positive for all $t>0$.

Proof. Assume that there exists $t_{0}=\inf \left\{t>0 \mid x_{2}(t)=\right.$ $0\}$. Based on the continuity $t_{0}>0$, it can be computed by evaluating the model system (2) at time $t_{0}$ :

$$
\dot{x}_{2}\left(t_{0}\right)= \begin{cases}r_{1} e^{-d \tau\left(z\left(t_{0}\right)\right)} \phi\left(t_{0}-\tau\left(z\left(t_{0}\right)\right)\right), & 0 \leq t_{0} \leq \widehat{\tau}, \\ r_{1} e^{-d \tau\left(z\left(t_{0}\right)\right)} x_{2}\left(t_{0}-\tau\left(z\left(t_{0}\right)\right)\right), & t_{0}>\widehat{\tau} .\end{cases}
$$

According to (6) and the initial conditions of model system (2), it is easy to show that $\dot{x}_{2}\left(t_{0}\right)>0$. On the other hand, it follows from the definition of $t_{0}$ that $\dot{x}_{2}\left(t_{0}\right)=0$, which is a contradiction. Consequently, $x_{2}(t)>0$ for all $t>0$.

Based on the positiveness of $x_{2}(t)$ and the third equation of model system (2), it is easy to show that $y(t)>0$ for $y(0)>$ $0, t>0$.

Consider the equation

$$
\begin{gathered}
\dot{u}(t)=-d u(t)-r_{1} e^{-d \tau(z)} x_{2}(t-\tau(z)), \\
u(0)=x_{1}(0)>0 .
\end{gathered}
$$

It is obvious to show that $\dot{u}(t)<0$; that is, $u(t)$ is strictly decreasing. By virtue of positiveness of $x_{2}(t), y(t)$, it derives that

$$
x_{1}(t)>u(t), \quad 0<t \leq \widehat{\tau} .
$$

By solving (7), it gives that

$$
u(t)=e^{-d t} u(0)-r_{1} e^{-d t} \int_{0}^{t} e^{d q} e^{-d \tau(z(q))} x_{2}(q-\tau(z(q))) d q .
$$

According to (5), it derives that

$$
\begin{aligned}
u(t)= & e^{-d t} \int_{-\widehat{\tau}}^{0} r_{1} e^{d s} \phi(s) d s \\
& -r_{1} e^{-d t} \int_{0}^{t} e^{d q} e^{-d \tau(z(q))} x_{2}(q-\tau(z(q))) d q .
\end{aligned}
$$

By substituting $p=q-\tau(z(q))$ in the above equation, it can be obtained that

$$
\begin{aligned}
u(t)= & r_{1} e^{-d t} \int_{-\widehat{\tau}}^{0} e^{d s} \phi(s) d s \\
& -r_{1} e^{-d t} \int_{-\widehat{\tau}}^{t-\tau(z(t))} \frac{e^{d p} x_{2}(p)}{1-\tau^{\prime}(z) \dot{z}(p)} d p
\end{aligned}
$$

which implies that

$$
\begin{aligned}
u(\widehat{\tau})= & r_{1} e^{-d \hat{\tau}} \int_{-\widehat{\tau}}^{0} e^{d s} \phi(s) d s \\
& -r_{1} e^{-d \hat{\tau}} \int_{-\widehat{\tau}}^{\hat{\tau}-\tau(z(\widehat{\tau}))} \frac{e^{d p} x_{2}(p)}{1-\tau^{\prime}(z) \dot{z}(p)} d p .
\end{aligned}
$$

According to $x_{2}(t)>0, y(t)>0$ for all $t>0$ and $t-$ $\tau(z(t))$ is an increasing function based on (H4). $1-\tau^{\prime}(z) \dot{z}(t)>$ 0 holds for $-\widehat{\tau} \leq t \leq \widehat{\tau}-\tau(z(\widehat{\tau}))$, and the following inequality can be obtained:

$$
u(\widehat{\tau}) \geq r_{1} e^{-d \hat{\tau}} \int_{-\widehat{\tau}}^{0} e^{d p} \phi(p)\left(1-\frac{1}{1-\tau^{\prime}(z) \dot{z}(p)}\right) d p>0
$$

since $u(\widehat{\tau})>0$ and $u(t)$ is strictly decreasing, $x_{1}(t)>0,-\widehat{\tau} \leq$ $t \leq 0$. By repeating this argument to include all positive time, it can be shown that $x_{1}(t)>0$ for all $t>0$. Hence, solutions of model system (2) with given initial conditions are positive for all $t>0$.

Theorem 2. If the hypotheses (H1)-(H4) hold and $r_{2}>q E$, all solutions of model system (2) are bounded within a region $\Omega$ :

$$
\begin{aligned}
\Omega=\{ & \left(x_{1}(t), x_{2}(t), y(t)\right) \mid \\
& \left.0<x_{1}(t)+x_{2}(t) \leq R_{1}, 0<y(t) \leq R_{2}\right\},
\end{aligned}
$$

where $R_{1}=\left(r_{1}+d\right)^{2} / 4 b d, R_{2}=\left(r_{2}-q E\right)\left(R_{1}+k_{2}\right) / a_{2}$.

Proof. Let $v(t)=x_{1}(t)+x_{2}(t)$, and it is easy to show that $v(t)>0$ based on positiveness of solutions of model system (2). By calculating the derivative of $v(t)$ along the solutions, it gives that

$$
\begin{aligned}
\dot{v}(t) & =r_{1} x_{2}(t)-d x_{1}(t)-b x_{2}^{2}(t)-\frac{a_{1} x_{2}(t) y(t)}{x_{2}(t)+k_{1}} \\
& <\left(r_{1}+d\right) x_{2}(t)-b x_{2}^{2}-d v(t) .
\end{aligned}
$$

By using the standard comparison principle in (15), it derives that

$$
\lim _{t \rightarrow \infty} \sup (v(t)) \leq \frac{\left(r_{1}+d\right)^{2}}{4 b d}=R_{1} .
$$


It follows from the third equation of model system (2) that

$$
\begin{aligned}
\dot{y}(t) & =\left(r_{2}-q E\right) y(t)-\frac{a_{2} y^{2}(t)}{x_{2}(t)+k_{2}} \\
& \leq\left(r_{2}-q E\right) y(t)-\frac{a_{2} y^{2}(t)}{R_{1}+k_{2}} .
\end{aligned}
$$

By utilizing the standard comparison principle in inequality (17) and $r_{2}-q E>0$, it gives that

$$
\lim _{t \rightarrow \infty} \sup y(t) \leq \frac{\left(r_{2}-q E\right)\left(R_{1}+k_{2}\right)}{a_{2}}=R_{2} \text {. }
$$

Consequently, all solutions of model system (2) are bounded within a region $\Omega$ :

$$
\begin{aligned}
\Omega= & \left\{\left(x_{1}(t), x_{2}(t), y(t)\right) \mid\right. \\
& \left.0<x_{1}(t)+x_{2}(t) \leq R_{1}, 0<y(t) \leq R_{2}\right\},
\end{aligned}
$$

where $R_{1}=\left(r_{1}+d\right)^{2} / 4 b d, R_{2}=\left(r_{2}-q E\right)\left(R_{1}+k_{2}\right) / a_{2}$.

\subsection{Existence of Equilibria and Uniqueness of Positive Equilib-} rium. The existence of biologically reasonable equilibria of model system (2) is investigated in this subsection. Since the biological interpretation of the positive equilibrium implies that immature prey, mature prey, and predator population all exist, uniqueness of positive equilibrium is also studied.

By simple computation, there are two equilibria denoted by $P_{0}(0,0,0)$ and $P_{1}\left(0,0, k_{2}\left(r_{2}-q E\right) / a_{2}\right)$. The biological interpretations of $P_{0}, P_{1}$ are as follows. For $P_{0}(0,0,0)$, it implies that all population in harvested prey predator ecosystem does not exist. For $P_{1}\left(0,0, k_{2}\left(r_{2}-q E\right) / a_{2}\right)$, it implies that there is not any predation source for predator population. It follows from the previous biological interpretations that population in such ecosystem cannot be maintained at an ideal level for sustainable development, which are not relevant to major investigation in this paper.

Furthermore, there is one or more positive equilibria denoted by $P^{*}\left(x_{1}^{*}, x_{2}^{*}, y^{*}\right)$. In order to discuss the existence of $P^{*}$, it is equivalent to show that the following equations always have at least one positive solution:

$$
\begin{gathered}
r_{1} x_{2}-d x_{1}-r_{1} e^{-d \tau\left(x_{1}+x_{2}+y\right)} x_{2}=0 \\
r_{1} e^{-d \tau\left(x_{1}+x_{2}+y\right)}-b x_{2}-\frac{a_{1} y}{x_{2}+k_{1}}=0 \\
r_{2}-q E-\frac{a_{2} y}{x_{2}+k_{2}}=0
\end{gathered}
$$

It follows from (20) that

$$
\begin{gathered}
y=\frac{\left(r_{2}-q E\right)\left(x_{2}+k_{2}\right)}{a_{2}}=f\left(x_{2}\right), \\
r_{1} x_{2}-d x_{1}=r_{1} x_{2} e^{-d \tau\left(x_{1}+g\left(x_{2}\right)\right)}, \\
r_{1} e^{-d \tau\left(x_{1}+g\left(x_{2}\right)\right)}=b x_{2}+a_{1} h\left(x_{2}\right),
\end{gathered}
$$

where $g\left(x_{2}\right)=x_{2}+y=x_{2}+f\left(x_{2}\right)$ and $h\left(x_{2}\right)=y /\left(x_{2}+k_{1}\right)=$ $f\left(x_{2}\right) /\left(x_{2}+k_{1}\right)$.
Theorem 3 (existence of positive equilibrium). Supposing that hypotheses (H1)-(H4) hold, if $k_{1} \geq k_{2}, r_{2}>q E$, and $a_{2} r_{1} e^{-d \tau_{1}}>a_{1} r_{2}$, then there exists at least one positive equilibrium $P^{*}$.

Proof. Let $\Gamma_{1}$ and $\Gamma_{2}$ be the solution curves of (22) and (23) for $x_{1} \geq 0, x_{2} \geq 0$, respectively. The analytical properties of curve $\Gamma_{1}$ and $\Gamma_{2}$ are as follows. $\Gamma_{1}$.

For $\Gamma_{1}$ : by simple computing, it can be found that $(0,0) \epsilon$

According to (H2) and positiveness of all solutions of model system (2), it is easy to show that $\lim _{x_{1} \rightarrow+\infty} \tau\left(x_{1}(t)+\right.$ $\left.g\left(x_{2}\right)\right)=\tau_{1}$, and

$$
\begin{aligned}
\lim _{x_{1} \rightarrow+\infty} x_{2}\left(x_{1}\right) & =\lim _{x_{1} \rightarrow+\infty} \frac{d x_{1}}{r_{1}\left(1-e^{-d \tau\left(x_{1}+g\left(x_{2}\right)\right.}\right)} \\
& =\lim _{x_{1} \rightarrow+\infty} \frac{d x_{1}}{r_{1}\left(1-e^{-d \tau_{1}}\right)}=+\infty .
\end{aligned}
$$

For $\Gamma_{2}$ : by differentiating $x_{2}$ against $x_{1}$ along $\Gamma_{2}$, it can be obtained that

$$
\begin{aligned}
\frac{d x_{2}}{d x_{1}}= & -d r_{1} e^{-d \tau\left(x_{1}+g\left(x_{2}\right)\right)} \\
& \times\left(d r_{1} e^{-d \tau\left(x_{1}+g\left(x_{2}\right)\right)} \tau^{\prime}\left(x_{1}+g\left(x_{2}\right)\right) \frac{a_{2}+r_{2}-q E}{a_{2}}\right. \\
& \left.+b+\frac{a_{1}\left(r_{2}-q E\right)\left(k_{1}-k_{2}\right)}{a_{2}\left(x_{2}+k_{1}\right)^{2}}\right)^{-1} .
\end{aligned}
$$

It can be shown that $\left(d x_{2} / d x_{1}\right)<0$, provided that $k_{1} \geq$ $k_{2}, r_{2}>q E$, and then $\Gamma_{2}$ is strictly decreasing.

Furthermore, according to $k_{1} \geq k_{2}, r_{2}>q E$, and $a_{2} r_{1} e^{-d \tau_{1}}>a_{1}\left(r_{2}-q E\right)$,

$$
\lim _{x_{1} \rightarrow \infty} x_{2}\left(x_{1}\right)=\frac{1}{b}\left(r_{1} e^{-d \tau_{1}}-\frac{a_{1}\left(r_{2}-q E\right)\left(x_{2}+k_{2}\right)}{a_{2}\left(x_{2}+k_{1}\right)}\right)>0 .
$$

Based on the above analysis, $\Gamma_{1}$ and $\Gamma_{2}$ intersect at some positive values, which proves the existence of positive equilibrium $P^{*}$.

Theorem 4 (uniqueness of positive equilibrium). Supposing that hypotheses (H1)-(H4) hold, if the following inequality holds

$$
\begin{aligned}
& 1- d \tau^{\prime}\left(x_{1}^{*}+g\left(x_{2}^{*}\right)\right)\left(b x_{2}^{*}+a_{1} h\left(x_{2}^{*}\right)\right) \\
& \times\left(a_{1} h^{\prime}\left(x_{2}^{*}\right)+d g^{\prime}\left(x_{2}^{*}\right) \tau^{\prime}\left(x_{1}^{*}+g\left(x_{2}^{*}\right)\right)\right. \\
&\left.\times\left(b x_{2}^{*}+a_{1} h\left(x_{2}^{*}\right)\right)\right)+a_{1} d h^{\prime}\left(x_{2}^{*}\right) \\
&+d \tau^{\prime}\left(x_{1}^{*}+g\left(x_{2}^{*}\right) g^{\prime}\left(x_{2}^{*}\right)\left(b x_{2}^{*}+a_{1} h\left(x_{2}^{*}\right)\right)\right. \\
& \times\left(d+b+d \tau^{\prime}\left(x_{1}^{*}+g\left(x_{2}^{*}\right)\right)\right)>0,
\end{aligned}
$$

then there exists a unique positive equilibrium. 
Proof. Based on (22) and (23), $x_{2}$ can be defined as the function of $x_{1}$ :

$$
\begin{aligned}
& \Gamma_{1}: x_{2}=g_{1}\left(x_{1}\right), \\
& \Gamma_{2}: x_{2}=g_{2}\left(x_{1}\right) .
\end{aligned}
$$

The positive equilibrium $P^{*}$ will be unique, provided that $g_{1}^{\prime}\left(x_{1}\right)>g_{2}^{\prime}\left(x_{1}\right)$ for every such $P^{*}$ otherwise reverse inequality holds.

By differentiating (22) with respect to $x_{1}$, it can be obtained that

$$
g_{1}^{\prime}\left(x_{1}\right)=\frac{d+r_{1} e^{-d \tau\left(x_{1}+g\left(x_{2}\right)\right)}\left(1-d \tau^{\prime}\left(x_{1}+g\left(x_{2}\right)\right)\right)}{r_{1}\left(1+e^{-d \tau\left(x_{1}+g\left(x_{2}\right)\right)} d \tau^{\prime}\left(x_{1}+g\left(x_{2}\right)\right) g^{\prime}\left(x_{2}\right)\right)} .
$$

By differentiating (23) with respect to $x_{1}$, it can be obtained that

$$
\begin{aligned}
& g_{2}^{\prime}\left(x_{1}\right) \\
& \quad=-\frac{b+d r_{1} \tau^{\prime}\left(x_{1}+g\left(x_{2}\right)\right) e^{-d \tau\left(x_{1}+g\left(x_{2}\right)\right)}}{a_{1} h^{\prime}\left(x_{2}\right)+d r_{1} g^{\prime}\left(x_{2}\right) \tau^{\prime}\left(x_{1}+g\left(x_{2}\right)\right) e^{-d \tau\left(x_{1}+g\left(x_{2}\right)\right)}} .
\end{aligned}
$$

On the other hand, some expressions about positive equilibrium $P^{*}\left(x_{1}^{*}, x_{2}^{*}, y^{*}\right)$ can be obtained based on (22) and (23),

$$
\begin{aligned}
& r_{1} x_{2}^{*}-d x_{1}^{*}=r_{1} x_{2}^{*} e^{-d \tau\left(x_{1}^{*}+g\left(x_{2}^{*}\right)\right)}, \\
& r_{1} e^{-d \tau\left(x_{1}^{*}+g\left(x_{2}^{*}\right)\right)}=b x_{2}^{*}+a_{1} h\left(x_{2}^{*}\right) .
\end{aligned}
$$

According to (31), $g_{1}^{\prime}\left(x_{1}^{*}\right)>g_{1}^{\prime}\left(x_{2}^{*}\right)$ is equivalent to the following inequality:

$$
\begin{aligned}
& 1- d \tau^{\prime}\left(x_{1}^{*}+g\left(x_{2}^{*}\right)\right)\left(b x_{2}^{*}+a_{1} h\left(x_{2}^{*}\right)\right) \\
& \times\left(a_{1} h^{\prime}\left(x_{2}^{*}\right)+d g^{\prime}\left(x_{2}^{*}\right) \tau^{\prime}\left(x_{1}^{*}+g\left(x_{2}^{*}\right)\right)\right. \\
&\left.\quad \times\left(b x_{2}^{*}+a_{1} h\left(x_{2}^{*}\right)\right)\right)+a_{1} d h^{\prime}\left(x_{2}^{*}\right) \\
&+d \tau^{\prime}\left(x_{1}^{*}+g\left(x_{2}^{*}\right) g^{\prime}\left(x_{2}^{*}\right)\right. \\
& \times\left(b x_{2}^{*}+a_{1} h\left(x_{2}^{*}\right)\right)\left(d+b+d \tau^{\prime}\left(x_{1}^{*}+g\left(x_{2}^{*}\right)\right)\right)>0 .
\end{aligned}
$$

This completes the proof.

3.3. Local Stability Analysis around Equilibria. Local stability of model system (2) around all equilibria of model system (2) is investigated. Furthermore, stability switch due to variation of maturity delay and harvest effort level is also studied in this subsection.

The characteristic equation of model system (2) about some equilibrium $\widetilde{P}=\left(\tilde{x}_{1}, \tilde{x}_{2}, \widetilde{y}\right)$ takes the following form:

$$
\left|\begin{array}{ccc}
\lambda+(d-\widetilde{A}) e^{-d \tau(\widetilde{z})} & r_{1}\left(1+e^{-(\lambda+d) \tau(\widetilde{z})}\right)-\widetilde{A} e^{-d \tau(\widetilde{z})} & -\widetilde{A} e^{-d \tau(\widetilde{z})} \\
\widetilde{A} e^{-d \tau(\tilde{z})} & \lambda+2 b \widetilde{x}+\frac{a_{1} k_{1} \tilde{y}}{\left(\tilde{x}_{2}+k_{1}\right)^{2}}+\widetilde{A} e^{-d \tau(\widetilde{z})}+r_{1} e^{-(\lambda+d) \tau(\widetilde{z})} & \widetilde{A} e^{-d \tau(\widetilde{z})}+\frac{a_{1} \tilde{x}_{2}}{k_{1}+\tilde{x}_{2}} \\
0 & -\frac{a_{2} \tilde{y}^{2}}{\left(k_{2}+\tilde{x}_{2}\right)^{2}} & \lambda-r_{2}+q E+\frac{2 a_{2} \tilde{y}}{k_{2}+\tilde{x}_{2}}
\end{array}\right|=0,
$$

where $\widetilde{A}=d r_{1} \tilde{x}_{2} \tau^{\prime}(\widetilde{z})$.

Theorem 5. Local stability analysis of model system (2) around $P_{0}$ and $P_{1}$ is as follows:

(a) if $r_{2}<q E$, then model system is locally stable around $P_{0}$, and $P_{1}$ is a saddle point which is unstable in the $y$ direction and stable in the $x_{1}-x_{2}$ plane;

(b) if $r_{2}>q E$, then model system is locally stable around $P_{1}$, and $P_{0}$ is a saddle point which is unstable in the $y$ direction and stable in the $x_{1}-x_{2}$ plane.

Proof. For $P_{0}(0,0,0),(33)$ reduces to

$$
\left(\lambda+d e^{-d \tau(0)}\right)\left(\lambda+r_{1} e^{-(\lambda+d) \tau(0)}\right)\left(\lambda-\left(r_{2}-q E\right)\right)=0 .
$$

By solving (34), it can be found that there are two negative eigenvalues and only one positive eigenvalue, provided $r_{2}>$ $q E$, which implies that $P_{0}$ is a saddle point which is unstable in the $y$-direction and stable in the $x_{1}-x_{2}$ plane. On the other hand, there are three negative eigenvalues, provided that $r_{2}<$ $q E$, which implies that $P_{0}$ is a stable point.

For $P_{1}\left(0,0,\left(k_{2}\left(r_{2}-q E\right) / a_{2}\right)\right),(33)$ reduces to

$$
\left(\lambda+d e^{-d \tau(\tilde{y})}\right)\left(\lambda+r_{1} e^{-(\lambda+d) \tau(\tilde{y})}+\frac{a_{1} \tilde{y}}{k_{1}}\right)\left(\lambda+\left(r_{2}-q E\right)\right)=0,
$$

where $\tilde{y}=k_{2}\left(r_{2}-q E\right) / a_{2}$. It follows from (35) that there are two negative eigenvalues and only one positive eigenvalue, provided $r_{2}<q E$, which implies that $P_{1}$ is a saddle point which is unstable in the $y$-direction and stable in the $x_{1}-x_{2}$ plane. On the other hand, there are three negative 
eigenvalues, provided that $r_{2}>q E$, which implies that $P_{1}$ is a stable point.
In order to discuss the local stability of model system (2) around the positive equilibrium $P^{*}\left(x_{1}^{*}, x_{2}^{*}, y^{*}\right),(33)$ reduces to

$$
\left|\begin{array}{ccc}
\lambda+d B^{*}-A^{*} & r_{1}\left(1+B^{*} e^{-\lambda \tau\left(z^{*}\right)}\right)-A^{*} & -A^{*} \\
A^{*} & \lambda+A^{*}+B^{*} e^{-\lambda \tau\left(z^{*}\right)}+2 b x_{2}^{*}+\frac{a_{1} k_{1} y^{*}}{\left(x_{2}^{*}+k_{1}\right)^{2}} & A^{*}+\frac{a_{1} x_{2}^{*}}{x_{2}^{*}+k_{1}} \\
0 & -\frac{a_{2} y^{* 2}}{\left(x_{2}^{*}+k_{2}\right)^{2}} & \lambda-r_{2}+q E+\frac{2 a_{2} y^{*}}{x_{2}^{*}+k_{2}}
\end{array}\right|=0
$$

where $A^{*}=d\left(r_{1} x_{2}^{*}-d x_{1}^{*}\right) \tau^{\prime}\left(z^{*}\right), B^{*}=\left(r_{1} x_{2}^{*}-d x_{1}^{*}\right) / r_{1} x_{2}^{*}$ and $z^{*}=x_{1}^{*}+x_{2}^{*}+y^{*}$.

It can be computed that

$$
M(\lambda)+N(\lambda) e^{-\lambda \tau\left(z^{*}\right)}=0,
$$

where $M(\lambda)=\lambda^{3}+m_{1} \lambda^{2}+m_{2} \lambda+m_{3}$ and $N(\lambda)=n_{1} \lambda^{2}+$ $n_{2} \lambda+n_{3}$

$$
\begin{aligned}
& m_{1}=d B^{*}+2 b x_{2}^{*}+\frac{a_{1} k_{1} y^{*}}{\left(x_{2}^{*}+k_{1}\right)^{2}}-r_{2}+q E+\frac{2 a_{2} y^{*}}{x_{2}^{*}+k_{2}}, \\
& m_{2}=\left(\frac{2 a_{2} y^{*}}{x_{2}^{*}+k_{2}}-r_{2}+q E\right)\left(A^{*}+2 b x_{2}^{*}+\frac{a_{1} k_{1} y^{*}}{\left(x_{2}^{*}+k_{1}\right)^{2}}\right) \\
& -A^{*}\left(r_{1}-A^{*}\right) \text {, } \\
& m_{3}=\frac{a_{2} y^{* 2} A^{* 2}}{\left(x_{2}^{*}+k_{2}\right)^{2}}+d B^{*}\left(A^{*}+2 b x_{2}^{*}+\frac{a_{1} k_{1} y^{*}}{\left(x_{2}^{*}+k_{1}\right)^{2}}\right) \\
& \times\left(\frac{2 a_{2} y^{*}}{x_{2}^{*}+k_{2}}-r_{2}+q E\right) \\
& -A^{*}\left(r_{1}+2 b x_{2}^{*}+\frac{a_{1} k_{1} y^{*}}{\left(x_{2}^{*}+k_{1}\right)^{2}}\right) \\
& \times\left(\frac{2 a_{2} y^{*}}{x_{2}^{*}+k_{2}}-r_{2}+q E\right), \\
& n_{1}=B^{*} \text {, } \\
& n_{2}=B^{*}\left(\frac{2 a_{2} y^{*}}{x_{2}^{*}+k_{2}}-r_{2}+q E-r_{1} A^{*}\right) \text {, } \\
& n_{3}=B^{*}\left(\frac{2 a_{2} y^{*}}{x_{2}^{*}+k_{2}}-r_{2}+q E\right)\left(d B^{*}-A^{*}-r_{1} A^{*}\right) \text {. }
\end{aligned}
$$

In the following part, dynamical behavior of model system (2) around the positive equilibrium $P^{*}$ is investigated. Furthermore, local stability analysis is discussed due to the variation of maturation delay and harvest effort level. By taking $\tau^{\prime}\left(z^{*}\right)$ as a bifurcation parameter, conditions for local stability switch are discussed with the increase of $\tau^{\prime}\left(z^{*}\right)$ from zero.
Case $1\left(\tau^{\prime}\left(z^{*}\right)=0\right)$. In the case of $\tau^{\prime}\left(z^{*}\right)=0$, it derives that $\tau\left(z^{*}\right)$ remains as a constant (zero or a positive constant) for all time $t>0$ based on (H2). In the following part, $\tau\left(z^{*}\right)$ is denoted as $\tau^{*}$ for simplifying. Furthermore, it can be computed that $A^{*}=0$, and $m_{i}, n_{i}(i=1,2,3)$ in (37) can be rewritten as follows:

$$
\begin{aligned}
& \widehat{m}_{1}=d B^{*}+2 b x_{2}^{*}+\frac{a_{1} k_{1} y^{*}}{\left(x_{2}^{*}+k_{1}\right)^{2}}-r_{2}+q E+\frac{2 a_{2} y^{*}}{x_{2}^{*}+k_{2}}, \\
& \widehat{m}_{2}=\left(\frac{2 a_{2} y^{*}}{x_{2}^{*}+k_{2}}-r_{2}+q E\right)\left(2 b x_{2}^{*}+\frac{a_{1} k_{1} y^{*}}{\left(x_{2}^{*}+k_{1}\right)^{2}}\right), \\
& \widehat{m}_{3}=d B^{*}\left(2 b x_{2}^{*}+\frac{a_{1} k_{1} y^{*}}{\left(x_{2}^{*}+k_{1}\right)^{2}}\right)\left(\frac{2 a_{2} y^{*}}{x_{2}^{*}+k_{2}}-r_{2}+q E\right), \\
& \widehat{n}_{1}=B^{*}, \\
& \widehat{n}_{2}=B^{*}\left(\frac{2 a_{2} y^{*}}{x_{2}^{*}+k_{2}}-r_{2}+q E\right), \\
& \widehat{n}_{3}=d B^{* 2}\left(\frac{2 a_{2} y^{*}}{x_{2}^{*}+k_{2}}-r_{2}+q E\right) .
\end{aligned}
$$

Theorem 6. Supposing that hypotheses (H1)-(H4) hold, if $r_{2}-$ $q E>0$, then model system (2) is stable around the positive equilibrium $P^{*}$ in the case of $\tau^{*}=0$.

Proof. When $\tau^{*}=0,(37)$ can be rewritten as follows:

$$
\lambda^{3}+\left(\widehat{m}_{1}+\widehat{n}_{1}\right) \lambda^{2}+\left(\widehat{m}_{2}+\widehat{n}_{2}\right) \lambda+\widehat{m}_{3}+\widehat{n}_{3}=0 .
$$

Based on the above analysis, it can be concluded that the roots of (40) have negative real parts by using the RouthHurwitz criteria [1]. Consequently, $P^{*}$ is locally stable in the case of $\tau^{*}=0$.

When $\tau^{*}>0$, let $\lambda=i \omega$ be a root of (37), where $\omega$ is positive. Substitute $\lambda=i \omega$ into (37) and separate the real and imaginary parts, and then two transcendental equations can be obtained as follows:

$$
\begin{gathered}
\omega^{3}-\widehat{m}_{2} \omega=\left(\widehat{n}_{1} \omega^{2}-\widehat{n}_{3}\right) \sin \left(\omega \tau^{*}\right)+\widehat{n}_{2} \omega \cos \left(\omega \tau^{*}\right), \\
\widehat{m}_{1} \omega^{2}-\widehat{m}_{3}=\widehat{n}_{2} \omega \sin \left(\omega \tau^{*}\right)-\left(\widehat{n}_{1} \omega^{2}-\widehat{n}_{3}\right) \cos \left(\omega \tau^{*}\right) .
\end{gathered}
$$


By squaring and adding (41), it can be obtained that

$$
\omega^{6}+B_{1} \omega^{4}+B_{2} \omega^{2}+B_{3}=0
$$

where $B_{1}=\widehat{m}_{1}^{2}-2 \widehat{m}_{2}-\widehat{n}_{1}^{2}, B_{2}=\widehat{m}_{2}^{2}-2 \widehat{m}_{1} \widehat{m}_{3}+2 \widehat{n}_{1} \widehat{n}_{3}-\widehat{n}_{2}^{2}$, $B_{3}=\widehat{m}_{3}^{2}-\widehat{n}_{3}^{2}$, and $\widehat{m}_{i}, \widehat{n}_{i}(i=1,2,3)$ have been defined in (40).

According to the values of $B_{i}(i=1,2,3)$ and the RouthHurwitz criteria [1], a simple assumption of the existence of a positive root for (42) is $B_{3}<0$.

If $B_{3}<0$ holds, then (42) has a positive root $\omega_{0}$, and (37) has a pair of purely imaginary roots of the form $\pm i \omega_{0}$. Consequently, it can be obtained by eliminating $\sin \left(\omega \tau^{*}\right)$ from (41):

$$
\begin{aligned}
& \cos \left(\omega \tau^{*}\right) \\
& =\frac{\left(\widehat{n}_{2}-\widehat{m}_{1} \widehat{n}_{1}\right) \omega^{4}+\left(\widehat{m}_{1} \widehat{n}_{3}+\widehat{m}_{3} \widehat{n}_{1}-\widehat{m}_{2} \widehat{n}_{2}\right) \omega^{2}-\widehat{m}_{3} \widehat{n}_{3}}{\left(\widehat{n}_{2} \omega^{2}\right)^{2}+\left(\widehat{n}_{3}-\widehat{n}_{1} \omega^{2}\right)^{2}},
\end{aligned}
$$

The $\tilde{\tau}_{k}$ corresponding to $\omega_{0}$ is as follows:

$$
\begin{aligned}
\widetilde{\tau}_{k}=\frac{1}{\omega_{0}} \arccos [ & \left(\left(\widehat{n}_{2}-\widehat{m}_{1} \widehat{n}_{1}\right) \omega^{4}\right. \\
& \left.+\left(\widehat{m}_{1} \widehat{n}_{3}+\widehat{m}_{3} \widehat{n}_{1}-\widehat{m}_{2} \widehat{n}_{2}\right) \omega^{2}-\widehat{m}_{3} \widehat{n}_{3}\right) \\
& \left.\times\left(\left(\widehat{n}_{2} \omega^{2}\right)^{2}+\left(\widehat{n}_{3}-\widehat{n}_{1} \omega^{2}\right)^{2}\right)^{-1}\right]+\frac{2 k \pi}{\omega_{0}}
\end{aligned}
$$

$k=0,1,2, \ldots$. By virtue of Butler's lemma [37], it can be concluded that the positive equilibrium $P^{*}$ remains locally stable for $\tau^{*}<\widetilde{\tau}_{0}$, as $k=0$.

Case $2\left(\tau^{\prime}\left(z^{*}\right)>0\right)$. In the case of $\tau^{\prime}\left(z^{*}\right)>0$, local stability of model system (2) around the positive equilibrium $P^{*}$ can change only if there exists at least one root of (37) such that $\operatorname{Re} \lambda=0$.

Let $\lambda=i v$ be one such root, where $\nu$ is positive. Substitute $\lambda=i v$ into (37) and separate the real and imaginary parts, and then two transcendental equations can be obtained as follows:

$$
\begin{aligned}
v^{3}-m_{2} v & =\left(n_{1} v^{2}-n_{3}\right) \sin \left(v \tau\left(z^{*}\right)\right) \\
& +n_{2} v \cos \left(v \tau\left(z^{*}\right)\right) \\
m_{1} v^{2}-m_{3}= & n_{2} v \sin \left(v \tau\left(z^{*}\right)\right) \\
& -\left(n_{1} v^{2}-n_{3}\right) \cos \left(v \tau\left(z^{*}\right)\right) .
\end{aligned}
$$

By squaring and adding (45), it can be obtained that

$$
v^{6}+C_{1} v^{4}+C_{2} v^{2}+C_{3}=0
$$

where $C_{1}=m_{1}^{2}-2 m_{2}-n_{1}^{2}, C_{2}=m_{2}^{2}-2 m_{1} m_{3}+2 n_{1} n_{3}-n_{2}^{2}$, $C_{3}=m_{3}^{2}-n_{3}^{2}$, and $m_{i}, n_{i}(i=1,2,3)$ have been defined in (37).
According to the values of $C_{i}(i=1,2,3)$ and the RouthHurwitz criteria [1], a simple assumption of the existence of a positive root for (42) is $C_{3}<0$, which derives that

$$
\begin{aligned}
& \tau^{\prime}\left(z^{*}\right) \\
& >\left(a_{1} k_{1} r_{1}\left(r_{2}-q E\right) x_{2}^{*}\left(x_{2}^{*}+k_{2}\right)\right. \\
& \left.+a_{2}\left(x_{2}^{*}+k_{1}\right)^{2}\left(r_{1} x_{2}^{*}\left(2 b x_{2}^{*}-1\right)+d x_{1}^{*}\right)\right) \\
& \times\left(r _ { 1 } x _ { 2 } ^ { * } \left[a_{1} k_{1} r_{1}\left(r_{2}-q E\right) x_{2}^{*}\left(x_{2}^{*}+k_{2}\right)\right.\right. \\
& +a_{2}\left(x_{2}^{*}+k_{1}\right)^{2} \\
& \left.\left.\times\left(d x_{1}^{*}(d+2)+r_{1} x_{2}^{*}\left(2 b x_{2}^{*}-d-1\right)\right)\right]\right)^{-1} .
\end{aligned}
$$

If the above inequality holds, then model system (2) is unstable around the positive equilibrium $P^{*}$ in the case of $\tau^{\prime}\left(z^{*}\right)>0$.

3.4. Global Stability Analysis of Positive Equilibrium. In this section, global stability of the positive equilibrium $P^{*}$ is discussed by using an iterative technique in the case of $\tau^{\prime}\left(z^{*}\right)=0$.

Lemma 7 (see [29]). Consider the following equation:

$$
\dot{x}=a x(t-\tau)-b x(t)-c x^{2}(t),
$$

where $a, b, c$, and $\tau$ are positive constants, and $x(t)>0$ for $t \in[-\tau, 0]$; it follows that

(i) If $a>b$, then $\lim _{t \rightarrow+\infty} x(t)=(a-b) / c$;

(ii) If $a<b$, then $\lim _{t \rightarrow+\infty} x(t)=0$.

Theorem 8. Supposing that hypotheses (H1)-(H4) and $r_{2}-$ $q E>0$ hold, if the following inequalities hold

$$
\begin{gathered}
b k_{2}+r_{1} e^{-d \tau^{*}}<a_{2} b y^{*}, \\
a_{2} k_{1} r_{1} e^{-d \tau^{*}}>a_{1}\left(r_{2}-q E\right)\left(R_{1}+k_{2}\right),
\end{gathered}
$$

then the positive equilibrium $P^{*}$ is globally asymptotically stable in the case of $\tau^{\prime}\left(z^{*}\right)=0$.

Proof. In the case of $\tau^{\prime}\left(z^{*}\right)=0$, it derives that $\tau\left(z^{*}\right)$ remains as a constant (zero or a positive constant) for all time $t>0$ based on (H2). In the following part, $\tau\left(z^{*}\right)$ is denoted as $\tau^{*}$ for simplifying. Let

$$
\begin{aligned}
U_{1} & =\lim _{t \rightarrow+\infty} \sup x_{2}(t), & V_{1} & =\lim _{t \rightarrow+\infty} \inf x_{2}(t), \\
U_{2} & =\lim _{t \rightarrow+\infty} \sup y(t), & V_{2} & =\lim _{t \rightarrow+\infty} \inf y(t) .
\end{aligned}
$$

In the following, we will claim that $U_{1}=V_{1}=x_{2}^{*}, U_{2}=$ $V_{2}=y^{*}$.

It follows from Theorem 2 that $x_{2}(t) \leq R_{1}\left(R_{1}\right.$ has been defined in Theorem 2). From model system (2),

$$
\dot{y}(t) \leq\left(r_{2}-q E\right) y(t)-\frac{a_{2} y^{2}(t)}{R_{1}+k_{2}} .
$$


By standard comparison argument, it derives that

$$
U_{2} \leq \frac{\left(r_{2}-q E\right)\left(R_{1}+k_{2}\right)}{a_{2}}:=J_{1}^{y},
$$

and then for sufficiently small $\epsilon>0$, there exists a $T_{11}>0$ such that if $t>T_{11}, y(t) \leq J_{1}^{y}+\epsilon$. Based on Theorem $1, x_{2}(t)+$ $k_{1}>k_{1}$, it can be obtained that for $t>T_{11}+\tau^{*}$,

$$
\begin{aligned}
\dot{x}_{2}(t) & \geq r_{1} e^{-d \tau^{*}} x_{2}\left(t-\tau^{*}\right)-b x_{2}^{2}(t)-\frac{a_{1}\left(J_{1}^{y}+\epsilon\right) x_{2}(t)}{x_{2}(t)+k_{1}} \\
& >r_{1} e^{-d \tau^{*}} x_{2}\left(t-\tau^{*}\right)-b x_{2}^{2}(t)-\frac{a_{1}\left(J_{1}^{y}+\epsilon\right) x_{2}(t)}{k_{1}} .
\end{aligned}
$$

Consider the following auxiliary equation:

$$
\dot{v}(t)=r_{1} e^{-d \tau^{*}} v\left(t-\tau^{*}\right)-b v^{2}(t)-\frac{a_{1}\left(J_{1}^{y}+\epsilon\right) v(t)}{k_{1}} .
$$

Under the condition $a_{2} k_{1} r_{1} e^{-d \tau^{*}}>a_{1}\left(r_{2}-q E\right)\left(R_{1}+k_{2}\right)$, it follows from Lemma 7 that

$$
\lim _{t \rightarrow+\infty} v(t)=\frac{k_{1} r_{1} e^{-d \tau^{*}}-a_{1}\left(J_{1}^{y}+\epsilon\right)}{b k_{1}}:=I_{1}^{x} .
$$

Hence, $V_{1} \geq I_{1}^{x}$. For sufficiently small $\epsilon>0$, there exits $T_{12} \geq T_{11}+\tau^{*}$ such that if $t>T_{22}$, then $x_{2}(t) \geq I_{1}^{x}-\epsilon$.

We derive from the model system (2) that for $t>T_{12}$,

$$
\dot{y}(t) \geq\left(r_{2}-q E\right) y(t)-\frac{a_{2} y^{2}(t)}{k_{2}+I_{1}^{x}-\epsilon} .
$$

A standard comparison argument shows that

$$
\lim _{t \rightarrow+\infty} y(t)=\frac{\left(r_{2}-q E\right)\left(k_{2}+I_{1}^{x}-\epsilon\right)}{a_{2}}:=I_{1}^{y} .
$$

Hence, for sufficiently small $\epsilon>0$, there is a $T_{21} \geq T_{12}$ satisfying if $t>T_{21}$, then $y(t) \geq I_{1}^{y}-\epsilon$. Consequently, for $t>T_{21}+\tau^{*}$,

$$
\dot{x}_{2}(t) \leq r_{1} e^{-d \tau^{*}} x_{2}\left(t-\tau^{*}\right)-b x_{2}^{2}(t)-\frac{a_{1}\left(I_{1}^{y}-\epsilon\right) x_{2}(t)}{R_{1}+k_{1}} .
$$

Consider the following auxiliary equation:

$$
\dot{v}(t)=r_{1} e^{-d \tau^{*}} v\left(t-\tau^{*}\right)-b v^{2}(t)-\frac{a_{1}\left(I_{1}^{y}-\epsilon\right) v(t)}{R_{1}+k_{1}} .
$$

It follows from Lemma 7 that

$$
\lim _{t \rightarrow+\infty} v(t)=\frac{\left(r_{1} e^{-d \tau^{*}}-b\right)\left(R_{1}+k_{1}\right)}{a_{1}\left(I_{1}^{y}-\epsilon\right)}:=J_{1}^{x} .
$$

Hence, $U_{1} \leq J_{1}^{x}$. For sufficiently small $\epsilon>0$, there exists a $T_{22} \geq T_{21}+\tau^{*}$ satisfying that if $t>T_{22}$, then $x_{2}(t) \leq J_{1}^{x}+\epsilon$. For $t>T_{22}$, it gives that

$$
\dot{y}(t) \leq\left(r_{2}-q E\right) y(t)-\frac{a_{2} y^{2}(t)}{k_{2}+J_{1}^{x}+\epsilon} .
$$

By standard comparison argument, it derives that

$$
\lim _{t \rightarrow+\infty} y(t)=\frac{\left(r_{2}-q E\right)\left(J_{1}^{x}+k_{2}+\epsilon\right)}{a_{2}}:=J_{2}^{y} .
$$

Hence, for sufficiently small $\epsilon>0$, there exists $T_{31} \geq T_{22}$ satisfying that if $t>T_{31}, y(t) \leq J_{2}^{y}+\epsilon$, the for $t>T_{31}+\tau^{*}$

$$
\dot{x}_{2}(t) \geq r_{1} e^{-d \tau^{*}} x_{2}\left(t-\tau^{*}\right)-b x_{2}^{2}(t)-\frac{a_{1}\left(J_{2}^{y}+\epsilon\right) x_{2}(t)}{k_{1}} .
$$

Consider the following auxiliary equation:

$\dot{v}(t)=r_{1} e^{-d \tau^{*}} v\left(t-\tau^{*}\right)-b v^{2}(t)-\frac{a_{1}\left(J_{2}^{y}+\epsilon\right) v(t)}{k_{1}}$.

By using Lemma 7, it can be obtained that

$$
\lim _{t \rightarrow+\infty} v(t)=\frac{k_{1} r_{1} e^{-d \tau^{*}}-a_{1}\left(J_{2}^{y}+\epsilon\right)}{b k_{1}}:=I_{2}^{x} .
$$

Since it is true for any sufficiently small $\epsilon>0, V_{1} \geq I_{2}^{x}$. Therefore, there exists $T_{32} \geq T_{31}+\tau^{*}$ such that if $t>T_{32}$, then $x_{2}(t) \geq I_{2}^{x}-\epsilon$.

It follows from model system (2) that for $t>T_{32}$,

$$
\dot{y}(t) \geq\left(r_{2}-q E\right) y(t)-\frac{a_{2} y^{2}(t)}{I_{2}^{x}-\epsilon+k_{2}} .
$$

By using standard comparison argument, it derives that

$$
\lim _{t \rightarrow+\infty} y(t)=\frac{\left(r_{2}-q E\right)\left(I_{2}^{x}-\epsilon+k_{2}\right)}{a_{2}}:=I_{2}^{y} .
$$

Since this is true for any sufficiently small $\epsilon>0, V_{2} \geq I_{2}^{y}$. Consequently, there exists $T_{41} \geq T_{32}$ satisfying if $t>T_{41}$, then $y(t) \geq I_{2}^{y}-\epsilon$.

It follows from model system (2) that for $t>T_{41}+\tau^{*}$,

$$
\dot{x}_{2}(t) \leq r_{1} e^{-d \tau^{*}} x_{2}\left(t-\tau^{*}\right)-b x_{2}^{2}(t)-\frac{a_{1}\left(I_{2}^{y}-\epsilon\right) x_{2}(t)}{R_{1}+k_{1}} .
$$

Consider the following auxiliary equation,

$\dot{v}(t) \leq r_{1} e^{-d \tau^{*}} v\left(t-\tau^{*}\right)-b v^{2}(t)-\frac{a_{1}\left(I_{2}^{y}-\epsilon\right) v(t)}{R_{1}+k_{1}}$.

By using Lemma 7, it derives that

$$
\lim _{t \rightarrow+\infty} x_{2}(t)=\frac{r_{1} e^{-d \tau^{*}}\left(R_{1}+k_{1}\right)-a_{1}\left(I_{2}^{y}-\epsilon\right)}{b\left(R_{1}+k_{1}\right)}:=J_{2}^{x} .
$$


Continuing the above process, four sequences $\left\{I_{n}^{x}\right\},\left\{I_{n}^{y}\right\}$, $\left\{J_{n}^{x}\right\},\left\{J_{n}^{y}\right\}, n=1,2, \ldots$, are obtained which take the following form

$$
\begin{aligned}
& J_{n}^{x}=\frac{r_{1} e^{-d \tau^{*}}\left(R_{1}+k_{1}\right)+a_{1} \epsilon-a_{1} I_{n}^{y}}{b\left(R_{1}+k_{1}\right)}, \\
& J_{n}^{y}=\frac{\left(\epsilon+k_{2}\right)\left(r_{2}-q E\right)+\left(r_{2}-q E\right) J_{n-1}^{x}}{a_{2}}, \\
& I_{n}^{x}=\frac{k_{1} r_{1} e^{-d \tau^{*}}-a_{1} \epsilon-a_{1} J_{n}^{y}}{b k_{1}}, \\
& I_{n}^{y}=\frac{\left(r_{2}-q E\right)\left(k_{2}-\epsilon\right)+\left(r_{2}-q E\right) I_{n}^{x}}{a_{2}} .
\end{aligned}
$$

It is easy to show that

$$
I_{n}^{x} \leq V_{1} \leq U_{1} \leq J_{n}^{x}, \quad I_{n}^{y} \leq V_{2} \leq U_{2} \leq J_{n}^{y} .
$$

By virtue of (71), it derives that

$$
\begin{aligned}
J_{n}^{y} & \\
= & \frac{\left(r_{2}-q E\right)\left[b\left(\epsilon+k_{2}\right)+r_{1} e^{-d \tau^{*}}\right]}{a_{2} b}+\frac{a_{1} \epsilon\left(r_{2}-q E\right)}{a_{2} b\left(R_{1}+k_{1}\right)} \\
& -\frac{a_{1}\left(r_{2}-q E\right)^{2}\left[k_{1}\left(r_{1} e^{-d \tau^{*}}+b k_{2}\right)+\epsilon\left(b k_{1}-a_{1}\right)-a_{1} J_{n-1}^{y}\right]}{a_{2}^{2} b^{2} k_{1}\left(R_{1}+k_{1}\right)} .
\end{aligned}
$$

Furthermore,

$$
\begin{aligned}
J_{n}^{y}- & J_{n-1}^{y} \\
& =\frac{\left(r_{2}-q E\right)\left[b\left(\epsilon+k_{2}\right)+r_{1} e^{-d \tau^{*}}\right]}{a_{2} b}+\frac{a_{1} \epsilon\left(r_{2}-q E\right)}{a_{2} b\left(R_{1}+k_{1}\right)} \\
& -\frac{a_{1}\left(r_{2}-q E\right)^{2}\left[k_{1}\left(r_{1} e^{-d \tau^{*}}+b k_{2}\right)+\epsilon\left(b k_{1}-a_{1}\right)-a_{1} J_{n-1}^{y}\right]}{a_{2}^{2} b^{2} k_{1}\left(R_{1}+k_{1}\right)} \\
& -J_{n-1}^{y} .
\end{aligned}
$$

If the following inequalities hold

$$
\begin{gathered}
b k_{2}+r_{1} e^{-d \tau^{*}}<a_{2} b y^{*}, \\
a_{2} k_{1} r_{1} e^{-d \tau^{*}}>a_{1}\left(r_{2}-q E\right)\left(R_{1}+k_{2}\right),
\end{gathered}
$$

then $J_{n}^{y}-J_{n-1}^{y} \leq 0$, which implies that $\left\{J_{n}^{y} \mid J_{n}^{y} \geq y^{*}, n=\right.$ $1,2, \ldots\}$ is monotonically decreasing. Hence, it can be shown that limitation of sequence $\left\{J_{n}^{y}\right\}$ exists. Taking $n \rightarrow+\infty$, it follows from (73) that

$$
\lim _{n \rightarrow+\infty} J_{n}^{y}=y^{*} .
$$

By using (71) and (76), it can be shown that

$$
\lim _{n \rightarrow+\infty} J_{n}^{x}=x_{2}^{*}, \quad \lim _{n \rightarrow+\infty} I_{n}^{y}=y^{*}, \quad \lim _{n \rightarrow+\infty} I_{n}^{x}=x_{2}^{*} .
$$

According to the definition of $U_{1}, U_{2}, V_{1}$, and $V_{2}$, it derives that

$$
U_{1}=V_{1}=x_{2}^{*}, \quad U_{2}=V_{2}=y^{*} .
$$

Hence,

$$
\lim _{t \rightarrow+\infty} x_{2}(t)=x_{2}^{*}, \quad \lim _{t \rightarrow+\infty} y(t)=y^{*} .
$$

Based on (5), it derives that

$$
x_{1}(t)=\int_{t-\widehat{\tau}}^{t} r_{1} e^{-d(t-s)} \phi(s) d s .
$$

By using L'Hospital's rule, it derives that

$$
\begin{aligned}
\lim _{t \rightarrow+\infty} x_{1}(t) & =\lim _{t \rightarrow+\infty} \frac{r_{1}\left[e^{d t} \phi(t)-e^{d(t-\widehat{\tau})} \phi(t-\widehat{\tau})\right]}{d e^{d t}} \\
& =\lim _{t \rightarrow+\infty} \frac{r_{1}}{d}\left[\phi(t)-e^{-d \hat{\tau}} \phi(t-\widehat{\tau})\right] \\
& =\lim _{t \rightarrow+\infty} \frac{r_{1}}{d}\left(1-e^{-d \hat{\tau}}\right) x_{2}^{*} .
\end{aligned}
$$

According to (20), it is easy to show that

$$
\lim _{t \rightarrow+\infty} x_{1}(t)=\lim _{t \rightarrow+\infty} \frac{r_{1}}{d}\left(1-e^{-d \hat{\tau}}\right) x_{2}^{*}=x_{1}^{*} .
$$

This completes the proof.

\section{Numerical Simulation}

With the help of MATLAB, numerical simulations are provided to understand the theoretical results which have been established in the previous sections of this paper. In order to facilitate the numerical simulation, it is assumed that $\tau(z(t))$ takes the following form [23]:

$$
\tau(z(t))=\tau_{0}+\tau_{m}-\tau_{m} e^{-z(t)},
$$

where $\tau_{m} \in\left(\tau_{0}, \tau_{1}\right)$ satisfying $\tau_{0}+\tau_{m}=\tau_{1}$. Based on Theorem 1, it follows from simple computation that

$$
\lim _{t \rightarrow 0} \tau(z(t))=\tau_{0}, \quad \lim _{t \rightarrow+\infty} \tau(z(t))=\tau_{1},
$$

which implies that $(\mathrm{H} 2)$ holds.

Values of parameters are taken from [24] which are used in Example 1 of [24] and set in appropriate units. $r_{1}=12$, $d=0.2, b=1.2, a_{1}=0.5, a_{2}=2, k_{1}=2, k_{2}=1$, $r_{2}=2, q=0.25$, and $E=4$. According to the given values of parameters, it follows from Theorems 3 and 4 that there exists a unique positive equilibrium $P^{*}(141.5454,5.6252,3.3126)$. Furthermore, it can be verified that $P^{*}$ is globally attractive based on Theorem 8 . Responses of model system (2) are indicated in Figure 1, and the phase portrait of model system (2) with different initial values is plotted in Figure 2. 


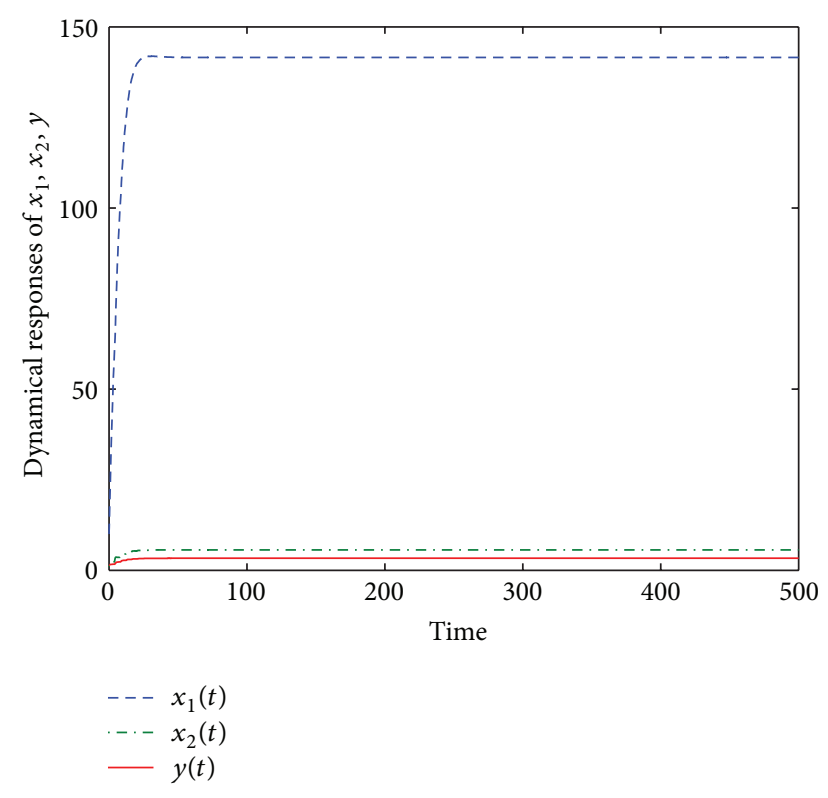

FIGURE 1: Dynamical responses of model system (2).

\section{Conclusion}

In this paper, a harvested prey predator model is proposed to investigate the effects of density-dependent maturation delay and harvest effort on the dynamics. Conditions which influence positiveness and boundedness of solutions of model system are obtained in Theorems 1 and 2, respectively. Existence of all equilibria of model system and uniqueness of the positive equilibrium are studied in Theorems 3 and 4, respectively. Biological interpretations of the positive equilibrium mean immature prey, mature prey, predator and harvest effort on predator population all exist in the harvested ecosystem. Consequently, we mainly concentrate on dynamical analysis around positive equilibrium in this paper. Local stability analysis in Theorem 6 reveals that local stability of the positive equilibrium loses due to variation of maturation delay and harvest effort level. Furthermore, global stability of the positive equilibrium is discussed by utilizing an iterative technique in Theorem 8 , which is utilized to investigate the coexistence and interaction mechanism of harvested prey-predator ecosystem.

Compared with the work done in [24] and the related work in [26], maturation delay for prey population in this paper relates to the density of all population within the harvested ecosystem, which accurately reflects the practical phenomena in the real world $[23,34-36]$. Furthermore, it should be noted that dynamics of prey predator model with density-dependent delay for predator population is investigated in [23], while dynamics of harvest effort on population within ecosystem is not considered. Compared with the work done in [23], harvest effort on predator population is introduced, and the effect of harvesting on model dynamics is also investigated in this paper. With the rapid development

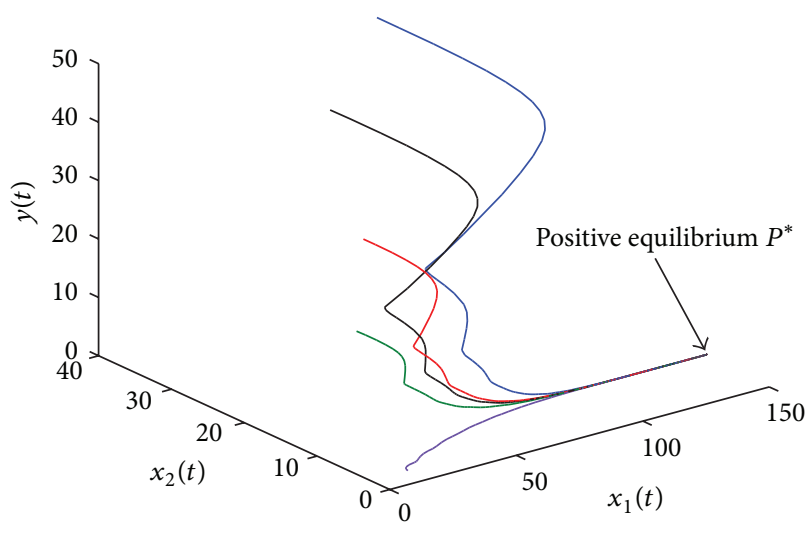

FIgUre 2: Phase portrait of model system (2) with different initial values.

of commercial harvesting on prey predator ecosystem in the real world, the introduction of harvest effort and related qualitative analysis makes the work done in this paper have some new and positive feature.

\section{Acknowledgments}

The authors gratefully acknowledge editors and anonymous reviewers' comments and patient work. This work is supported by National Natural Science Foundation of China, grant No. 61104003, grant No. 61273008 and grant No. 61104093. Research Foundation for Doctoral Program of Higher Education of Education Ministry, grant No. 20110042120016. Hebei Province Natural Science Foundation, grant No. F2011501023. Fundamental Research Funds for the Central Universities, grant No. N120423009. Research Foundation for Science and Technology Pillar Program of Northeastern University at Qinhuangdao, grant No. XNK201301. This work is supported by State Key Laboratory of Integrated Automation of Process Industry, Northeastern University, supported by Hong Kong Admission Scheme for Mainland Talents and Professionals, Hong Kong Special Administrative Region.

\section{References}

[1] M. Kot, Elements of Mathematical Ecology, Cambridge University Press, Cambridge, UK, 2001.

[2] W. G. Aiello and H. I. Freedman, "A time-delay model of singlespecies growth with stage structure," Mathematical Biosciences, vol. 101, no. 2, pp. 139-153, 1990.

[3] W. G. Aiello, H. I. Freedman, and J. Wu, "Analysis of a model representing stage-structured population growth with statedependent time delay," SIAM Journal on Applied Mathematics, vol. 52, no. 3, pp. 855-869, 1992.

[4] Y. Kuang, Delay Differential Equations with Applications in Population Dynamics, vol. 191, Academic Press, San Diego, Calif, USA, 1993.

[5] J. K. Hale and S. Lunel, Introduction to Functional Differentail Equations, Springer, New York, NY, USA, 1993. 
[6] O. Arino, E. Sanchez, and A. Fathallah, "State-dependent delay differential equations in population dynamics: modeling and analysis," in Fields Institute Communications, vol. 29, pp. 19-36, American Mathematical Society, Providence, RI, USA, 2001.

[7] J. Al-Omari and S. A. Gourley, "Monotone travelling fronts in an age-structured reaction-diffusion model of a single species," Journal of Mathematical Biology, vol. 45, no. 4, pp. 294-312, 2002.

[8] R. Xu, M. A. J. Chaplain, and F. A. Davidson, "Persistence and stability of a stage-structured predator-prey model with time delays," Applied Mathematics and Computation, vol. 150, no. 1, pp. 259-277, 2004.

[9] J. Cui and X. Song, "Permanence of predator-prey system with stage structure," Discrete and Continuous Dynamical Systems B, vol. 4, no. 3, pp. 547-554, 2004.

[10] S. A. Gourley and Y. Kuang, "A stage structured predator-prey model and its dependence on maturation delay and death rate," Journal of Mathematical Biology, vol. 49, no. 2, pp. 188-200, 2004.

[11] M. Bandyopadhyay and S. Banerjee, "A stage-structured preypredator model with discrete time delay," Applied Mathematics and Computation, vol. 182, no. 2, pp. 1385-1398, 2006.

[12] X.-P. Yan and W.-T. Li, "Hopf bifurcation and global periodic solutions in a delayed predator-prey system," Applied Mathematics and Computation, vol. 177, no. 1, pp. 427-445, 2006.

[13] X. Song and H. Guo, "Global stability of a stage-structured predator-prey system," International Journal of Biomathematics, vol. 1, no. 3, pp. 313-326, 2008.

[14] H. Zhang, P. Georgescu, and L. Chen, "An impulsive predatorprey system with Beddington-DeAngelis functional response and time delay," International Journal of Biomathematics, vol. 1, no. 1, pp. 1-17, 2008.

[15] R. Xu and Z. Ma, "Stability and Hopf bifurcation in a predatorprey model with stage structure for the predator," Nonlinear Analysis. Real World Applications, vol. 9, no. 4, pp. 1444-1460, 2008.

[16] R. Xu and Z. Ma, "Stability and Hopf bifurcation in a ratiodependent predator-prey system with stage structure," Chaos, Solitons \& Fractals, vol. 38, no. 3, pp. 669-684, 2008.

[17] S. Gao, L. Chen, and Z. Teng, "Hopf bifurcation and global stability for a delayed predator-prey system with stage structure for predator," Applied Mathematics and Computation, vol. 202, no. 2, pp. 721-729, 2008.

[18] X.-P. Yan and C.-H. Zhang, "Hopf bifurcation in a delayed Lokta-Volterra predator-prey system," Nonlinear Analysis. Real World Applications, vol. 9, no. 1, pp. 114-127, 2008.

[19] R. Shi and L. Chen, "The study of a ratio-dependent predatorprey model with stage structure in the prey," Nonlinear Dynamics, vol. 58, no. 1-2, pp. 443-451, 2009.

[20] Z.-P. Ma, H.-F. Huo, and C.-Y. Liu, "Stability and Hopf bifurcation analysis on a predator-prey model with discrete and distributed delays," Nonlinear Analysis. Real World Applications, vol. 10, no. 2, pp. 1160-1172, 2009.

[21] J. Liu and T. Zhang, "Hopf bifurcation and stability analysis for a stage-structured system," International Journal of Biomathematics, vol. 3, no. 1, pp. 21-41, 2010.

[22] R. Xu, "Global dynamics of a predator-prey model with time delay and stage structure for the prey," Nonlinear Analysis. Real World Applications, vol. 12, no. 4, pp. 2151-2162, 2011.

[23] M. Agarwal and S. Devi, "A stage-structured predator-prey model with density-dependent maturation delay," International Journal of Biomathematics, vol. 4, no. 3, pp. 289-312, 2011.
[24] H.-F. Huo, X. Wang, and C. Castillo-Chavez, "Dynamics of a stage-structured Leslie-Gower predator-prey model," Mathematical Problems in Engineering, vol. 2011, Article ID 149341, 22 pages, 2011.

[25] L. Zha, J.-A. Cui, and X. Zhou, "Ratio-dependent predatorprey model with stage structure and time delay," International Journal of Biomathematics, vol. 5, no. 4, Article ID 1250014, 2012.

[26] Z. Li, M. Han, and F. Chen, "Global stability of a stagestructured predator-prey model with modified Leslie-Gower and Holling-type II schemes," International Journal of Biomathematics, vol. 5, no. 6, Article ID 1250057, 2012.

[27] K. Gapalsamy, Stability and Oscillations in Delay Equations of Population Dynamics, Kluwer Academic, London, UK, 1992.

[28] X. Zhang, L. Chen, and A. U. Neumann, “The stage-structured predator-prey model and optimal harvesting policy," Mathematical Biosciences, vol. 168, no. 2, pp. 201-210, 2000.

[29] X. Song and L. Chen, "Optimal harvesting and stability for a two-species competitive system with stage structure," Mathematical Biosciences, vol. 170, no. 2, pp. 173-186, 2001.

[30] T. K. Kar, "Selective harvesting in a prey-predator fishery with time delay," Mathematical and Computer Modelling, vol. 38, no. 3-4, pp. 449-458, 2003.

[31] T. K. Kar and U. K. Pahari, "Modelling and analysis of a preypredator system with stage-structure and harvesting," Nonlinear Analysis. Real World Applications, vol. 8, no. 2, pp. 601-609, 2007.

[32] T. K. Kar and K. Chakraborty, "Effort dynamic in a preypredator model with harvesting," International Journal of Information \& Systems Sciences, vol. 6, no. 3, pp. 318-332, 2010.

[33] T. K. Kar and A. Ghorai, "Dynamic behaviour of a delayed predator-prey model with harvesting," Applied Mathematics and Computation, vol. 217, no. 22, pp. 9085-9104, 2011.

[34] R. Gambell, W. N. Bonner, and D. W. H. Walton, Birds and Mammals-Antarctic Whales, in Antarctica, Pergamon, New York, NY, USA, 1985.

[35] J. M. Jeschke, "Density-dependent effects of prey defenses and predator offenses," Journal of Theoretical Biology, vol. 242, no. 4, pp. 900-907, 2006.

[36] H. Li and Y. Takeuchi, "Dynamics of the density dependent predator-prey system with Beddington-DeAngelis functional response," Journal of Mathematical Analysis and Applications, vol. 374, no. 2, pp. 644-654, 2011.

[37] B. D. Hassard, N. D. Kazarinoff, and Y. H. Wan, Theory and Applications of Hopf Bifurcation, vol. 41, Cambridge University Press, Cambridge, UK, 1981. 


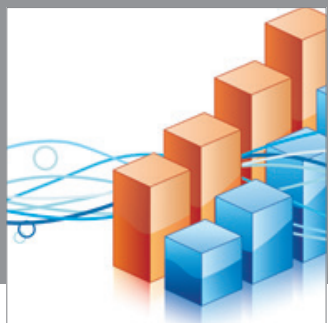

Advances in

Operations Research

mansans

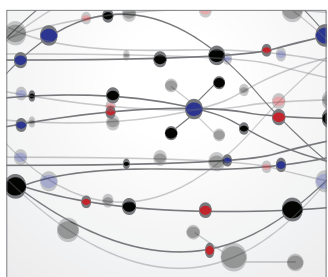

The Scientific World Journal
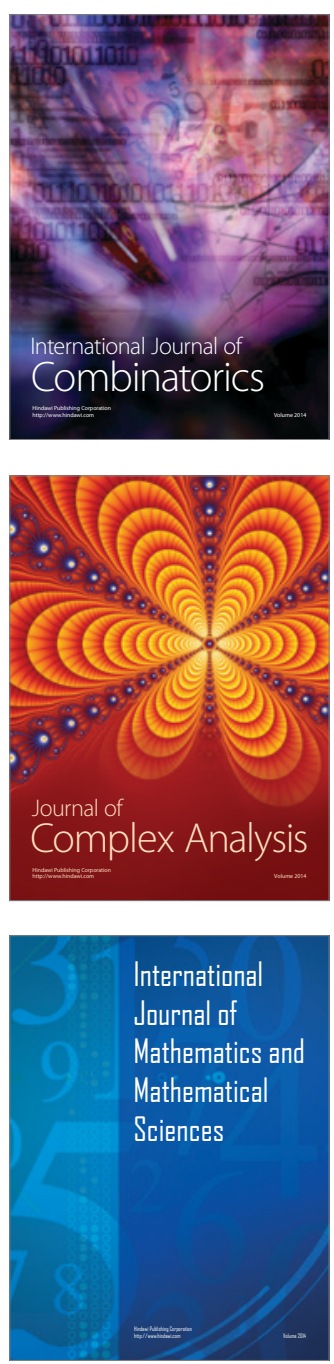
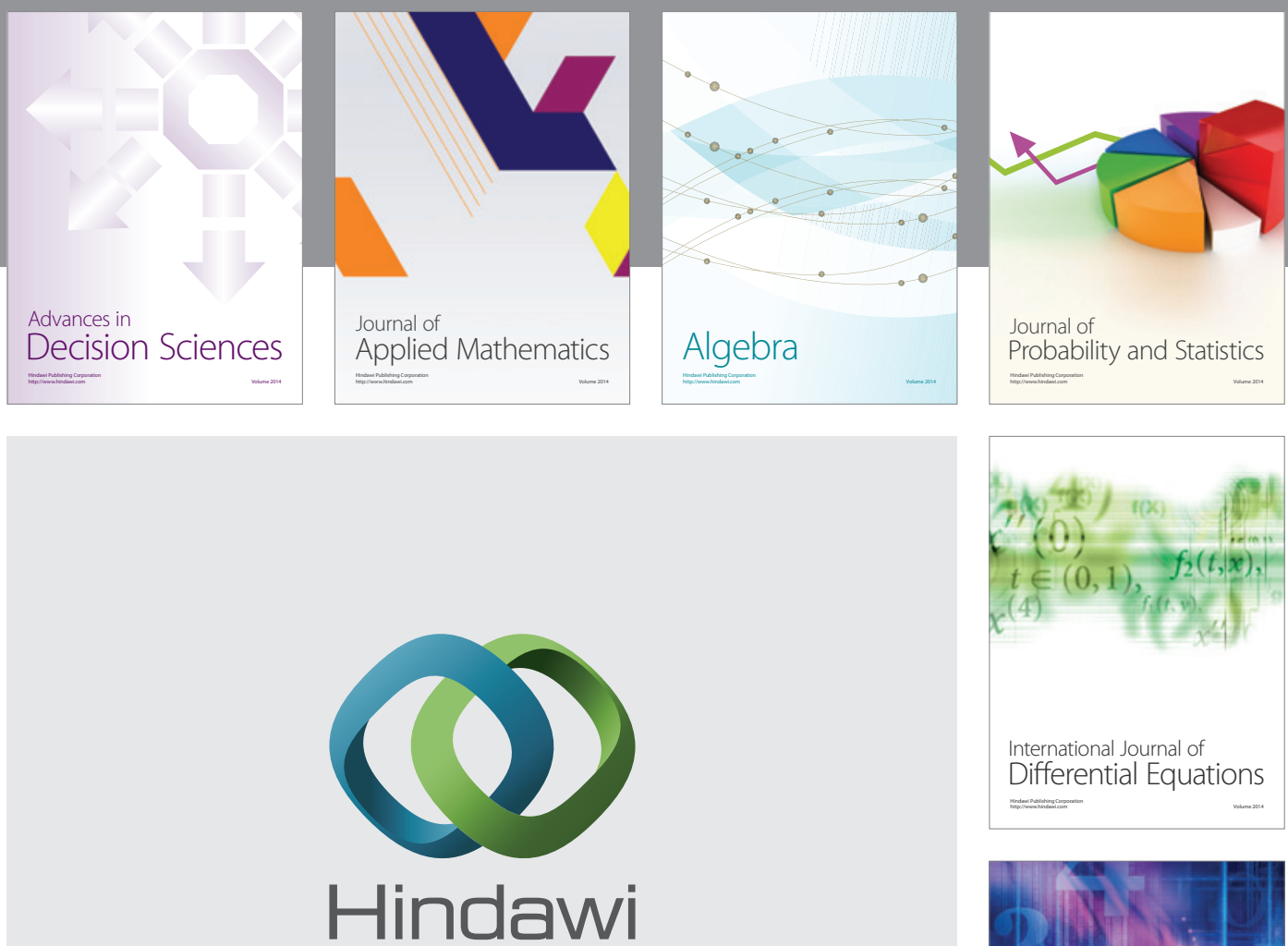

Submit your manuscripts at http://www.hindawi.com
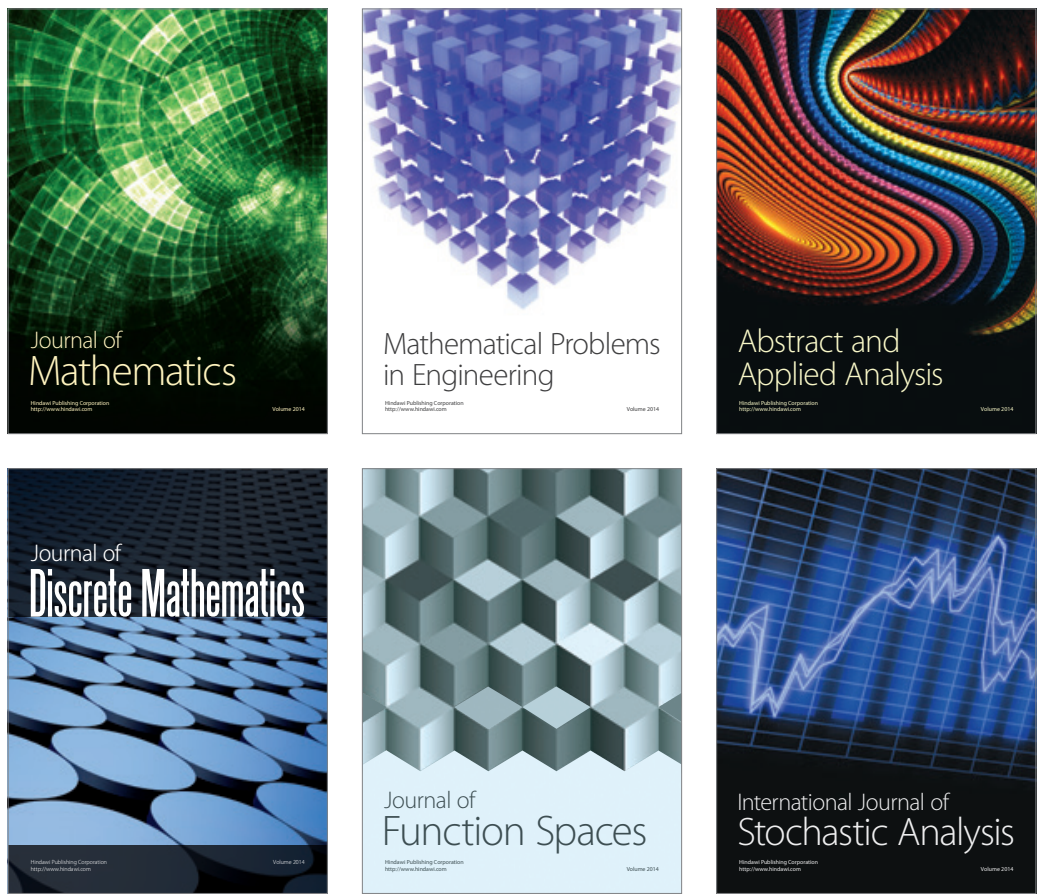

Journal of

Function Spaces

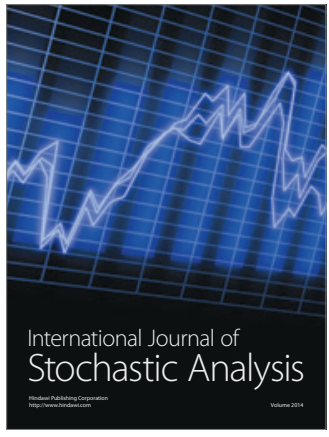

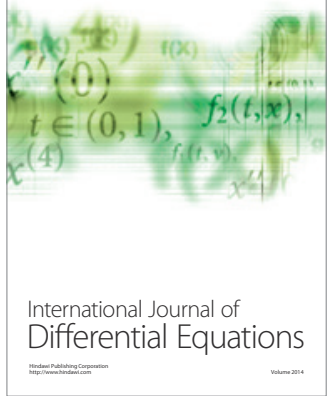
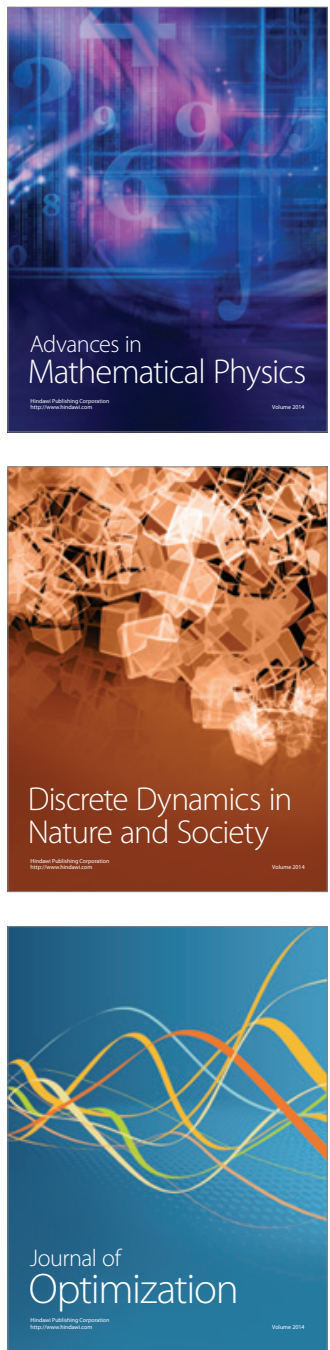